\title{
Camellia Sinensis Leaves Hydroalcoholic Extract Improves the Alzheimer's Disease-Like Alterations Induced by Type 2 Diabetes in Rats
}

\author{
Muhammet Emin Cam ${ }^{1,2,3}$ (D), Turgut Taskin ${ }^{4}$ (D) \\ ${ }^{1}$ Marmara University, Faculty of Pharmacy, Department of Pharmacology, Istanbul, Turkey. \\ ${ }^{2}$ Marmara University, Center for Nanotechnology and Biomaterials Research, Istanbul, Turkey. \\ ${ }^{3}$ University College London, Department of Mechanical Engineering, London, UK. \\ ${ }^{4}$ Marmara University, Faculty of Pharmacy, Department of Pharmacognosy, Istanbul, Turkey. \\ Correspondence Author: Muhammet Emin Cam \\ E-mail: muhammet.cam@marmara.edu.tr \\ Received: $06.02 .2020 \quad$ Accepted: 21.03. 2020
}

\begin{abstract}
Objective: Novel investigations have confirmed that hyperglycemia is strictly associated with the development of cognitive impairment and dementia. Sodium-dependent glucose transporter (SGLT) inhibitors, which are oral antidiabetic drugs, are currently being investigated as the medication in Alzheimer's disease (AD). In our study, Camellia sinensis (green tea), which inhibits sodium-dependent glucose transporter-1 (SGLT-1), was used in the treatment of type 2 diabetes mellitus (T2DM)-induced AD-like alterations via its antidiabetic effects.

Methods: High-fat diet/streptozotocin-treated rat model was chosen to provide T2DM-induced AD-like alterations. Antidiabetic effects were evaluated with the measurement of blood glucose level (BGL), oral glucose tolerance test (OGTT), and insulin tolerance test (ITT). On the other hand, novel object recognition test (NORT), open field test (OFT), passive avoidance test (PAT), and Morris's water maze (MWM) test were performed to investigate the anti-Alzheimer's effects of $C$. Sinensis.

Results: $C$. sinensis tolerated BGL for a short time but metformin, the first medication prescribed for T2DM, tolerated BGL during the test for $120 \mathrm{~min}$. C. sinensis increased the number of square crosses and the frequency of grooming activity in a similar manner to metformin in OFT. C. sinensis treatment improved exploratory behavior and memory retention components in NORT. The step-through latency decreased in HFD/ STZ-treated rat model but it improved with metformin and $C$. sinensis treatment in PAT. According to the results obtained by the MWM test, $C$. sinensis treatment slightly improved learning.
\end{abstract}

Conclusion: $C$. sinensis improved short-term memory and increased the locomotor activity in rats according to the results obtained by NORT, $\mathrm{OFT}$, and PA.

Keywords: Camellia sinensis, sodium-dependent glucose transporter, diabetes mellitus, Alzheimer's disease, memory

\section{INTRODUCTION}

Alzheimer's disease (AD), a progressive brain disorder, is the most common form of dementia among older adults. (1) An estimated 6 million Americans of all ages are living with $A D$ in 2019. Out of the total USA population, one in 10 people at the age of 65 and older has AD. This disease is becoming a menace to aging population all over the world and it is expected to has 13-20 million $A D$ cases in the next three decades. (2-4)

According to studies, $A D$ is characterized with the occurrence of loss of synapses, changes in neurotransmitter expression, reduced neutrophil numbers, synaptotoxicity, extracellular amyloid- $\beta(A \beta)$ plaques and neurofibrillary tangles in the intracellular environment, neuronal death and neural atrophy in the final phase. Beta-amyloid plaques may contribute to cell death by interfering with neuron-to-neuron communication at synapses, while tau tangles block the transport of nutrients and other essential molecules inside neurons. Actually this differances that occurs with damaging and destroying of neuron cells in the brain are thought to began 20 years or more before symptoms arise with small changes that are unnoticeable to the person affected. $(3,5-8)$

When the first stage of the onset of Alzheimer's appearance, the brain can initially compensate it, but as the damage to nerve cells continues, the brain can no longer compensate for the changes and AD causes to loss intellectual and social skills of people by affecting their ability such as thinking, speaking, learning, memory, walking, swallowing, resuming daily activities, planning family events or participating in sports. When patients reach the final stages of Alzheimer's disease, they become bed-bound and require 24-hour care. Alzheimer's disease is ultimately fatal. $(3,9)$

In some studies $A D$ is found to be associated with multiple risk factors such as aging, psychosocial (e.g., low educational level, lack of social engagement, and poor social 
networking), genetic (family history), genetic mutations (the extra copy of chromosome 21 that characterizes Down syndrome), traumatic brain injury, carrying the e4 form of the apolipoprotein E (APOE) gene, vascular parameters (e.g., obesity, tobacco use, and blood cholesterol) and vascular diseases (e.g., diabetes mellitus, hypertension, and stroke). $(5,8,10,11)$

Type 2 Diabetes Mellitus (T2DM), characterized by hyperglycemia, insulin resistance, and impaired insulin sensitivity. T2DM accounts for $90-95 \%$ of all diabetes by affecting over 300 million people worldwide. T2DM causes various serious complications of heart, eyes, nerves, liver and kidneys and is associated with decrements in cognitive function and changes in brain structure. Normal brain function is further compromised by the decreased ability of the brain to metabolize glucose that is main fuel of brain. (1214)

In in vitro, in vivo and human clinical studies, after the appearance of T2DM, T2DM has been found to be effective in the initiation and progression of $A D$ with common biological mechanisms directly or as cofactor. These biological mechanisms are insulin resistance, impaired glucose metabolism, $\beta$-amyloid formation, oxidative stress and the presence of advanced glycation and products. (15) It is thought that AD occurs when a similar cellular insulin resistance and insulin deficiency process occurs in the brain. (16)

In literature, it has been shown that high-fat diet (HFD) is a common risk factor for T2DM and AD. Therefore, HFD/ streptozotocin (STZ)-treated rat models were used to provide T2DM-induced AD-like alterations in some studies and also in our study. (17-19)

$A D$ treatment is divided into pharmacological and nonpharmacological, but both treatments do not slow or stop the damage and destruction of neurons that cause AD symptoms. The pharmacological treatment of rivastigmine, galantamine, donepezil, memantine, donepezil and tacrin combined with memantine treatment is approved by the US Food and Drug Administration (FDA) and this treatment includes vitamins C, D and E, omega-3 fatty acids and ginkgo biloba for use as complementary therapy. Also, some plant extracts are used and found effective in the treatment of AD. (20-23) Nonpharmacological treatment includes computerized memory training, listening to your favorite music and special lighting to reduce sleep disturbances. $(11,24)$

Moreover, a sufficient medical treatment targeting $A D$ in T2DM patients has not yet been proved. To use antidiabetic drugs such as metformin in order to treat $A D$ is an alternative treatment strategy and has been given more importance in the last decade. (25) The possible mechanisms of green tea for decreasing blood glucose level are including the inhibition of $\alpha$-glucosidase activity, intestinal sodium-glucose cotransporter-1 (SGLT-1) and glucose transport-2. In our study, a plant extract prepared by Camellia sinensis (green tea) was used in the treatment of T2DM-induced AD-like alterations via its SGLT-1 inhibitor effects. $(26,27,28)$

Camellia sinensis that is a member of the Theaceae family, is the second most consumed beverage in the world, after water. This plant native to China and Southeast Asia. (29) The leaves of $C$. sinensis are dark green, alternate and oval, with serrated edges, and the blossoms are white, fragrant, and appear in clusters or singly. This plant can reach to the heights of 30 feet, but they are usually pruned to 2-5 feet for growing. (30) It has 3 different varieties: Green tea, black tea, and oolong tea. These are made from the same plant but are processed differently, depending on their degree of fermentation. (31)

Chemical components of $70 \%$ ethanol extract of $C$. sinensis are polyphenols, caffeine, flavonoids, catechins such as catechin, gallocatechin, epigallocatechin, epicatechin, epigallocatechin gallate, gallocatechin gallate, epicatechin gallate, and catechin gallate. (32)

These chemical compounds show some medicinal effects such as hepatoprotective, cardioprotective, neuroprotective, anticancer, antiobesity, antidiabetic, antibacterial, and antiviral. (33) Furthermore in some studies, C. sinensis is thought to be effective in the treatment of $A D$ with its antineurodegenerative effects. $(31,34-36)$

In this study, we aimed to investigate anti-Alzheimer's effect of $C$. sinensis in T2DM-induced AD model via its antidiabetic effects. Antidiabetic effects were evaluated with the measurement of blood glucose level (BGL), oral glucose tolerance test (OGTT), and insulin tolerance test (ITT). On the other hand, novel object recognition test (NORT), open field test (OFT), passive avoidance test (PAT), and Morris's water maze (MWM) test were performed to investigate the antiAlzheimer's effects of $C$. Sinensis.

\section{MATERIALS AND METHOD}

\subsection{Materials}

Streptozotocin (STZ) and glucose were purchased from Santa Cruz Biotechnology, Inc (Dallas, TX, USA). High-fat diet (HFD) was obtained from MFD Company and it contains as percentage of calories $58 \%$ fat. Insulin glarjin (Lantus ${ }^{\circledR}$ ) was bought from Sanofi Aventis.

\subsection{Plant material and extraction}

The methanolic extract of $C$. sinensis leaves were given to the rats in this study. $C$. sinensis was obtained from local market and identified by Assist. Prof. Dr. İsmail Şenkardeş, Marmara University, Faculty of Pharmacy, Pharmaceutical Botany Department. The leaves of $C$. sinensis was extracted using methanol: water $(70: 30, \mathrm{v} / \mathrm{v})$ solvent by maceration for 7 days. After extraction, the sample was filtered using filter paper, then solvent was evaporated using rotary evaporator. The crude extract was stored in $4{ }^{\circ} \mathrm{C}$ to use in the experiment. 


\subsection{In vivo animal test}

In vivo animal test were perfomed with the permission of Marmara University Animal Experiments Local Ethics Committee (permission number: 77.2018.mar). Adult female and male Sprague dawley rats at the age of 3-4 month and weighting 250 to $300 \mathrm{~g}$ were obtained from Marmara University Experimental Animal Implementation and Research Center (DEHAMER). The rats were maintained in controlled temperature $\left(20 \pm 2{ }^{\circ} \mathrm{C}\right)$, humidity $(40-60 \%)$ and $12 \mathrm{~h}$ dark/light cycle)-regulated rooms. All experiments were performed at the fixed hours between 09:00-12:00. Water were provided ad libitum to all groups. However, HFD was given to all groups except of control group. All necessary precautions were taken before the experiment and the factors that would adversely affect the parameters were minimized during the study. All rats were kept in their cages for a week to adapt conditions before starting the behavioral tests.

\subsubsection{Experimental scheme}

There are 4 groups in the animal test and 12 animals in each test group. $1^{\text {st }}$ group is control group (C) and distilled water $\left(5 \mathrm{ml} / \mathrm{kg}\right.$ ) was given intragastrically to healty rats. $2^{\text {nd }}$ group is Alzheimer's disease group (AD) and T2DM-induced AD model was applied to the rats such as $3^{\text {rd }}$ and $4^{\text {th }}$ groups. However, only distilled water was given to $2^{\text {nd }}$ group but $C$. Sinensis $(625$ $\mathrm{mg} / \mathrm{kg}$ ) (37) and metformin (400 mg/kg) (13) treatments were given intragastrically for 22 days to the $3^{\text {rd }}(A D+C S)$ and $4^{\text {th }}$ groups ( $A D+M$, positive control group), respectively. Metformin and C. Sinensis were given in distilled water $(5 \mathrm{ml} /$ $\mathrm{kg})$.

\subsubsection{Induction of T2DM-induced $A D$}

Rats were administered a HFD ( $58 \%$ kcal fat) for 8 weeks. Rats fed HFD were injected once at week 4 with a low dose of STZ (40 mg/kg, solved in $0.1 \mathrm{M}$ citrate buffer, $\mathrm{pH}: 4.5$ ) to shorten the time taken for the animal model to be established by inducing partial insulin deficiency. One week after the injection, BGLs were measured using a glucometer (Contour Plus, Bayer Diagnostics) and the rats with BGL over $200 \mathrm{mg} /$ $\mathrm{dL}$ were classified as T2DM (13). All treatments were started after STZ injection.

\subsection{Determination of body weight and BGL}

Body weight (b.w.) and BGL of all groups were measured weekly and blood samples were taken from the tail vein to monitore BGL using a glucometer.

\subsection{Oral glucose tolerance test (OGTT)}

Glucose tolerance test is a commonly used clinical test to diagnose glucose intolerance and T2DM. After $12 \mathrm{~h}$ of fasting, treatments were applied and a single dose of glucose $(2 \mathrm{~g} / \mathrm{kg}, \mathrm{b} . \mathrm{w}$.) was given by gavage 30 minutes later. Glucose concentrations were monitored in the blood collected from the tail vein at $0,30,60$, and 120 min following the glucose injection. (14)

\subsection{Insulin tolerance test (ITT)}

Rats were fasted for $12 \mathrm{~h}$ before the test and they were injected with insulin ( $1 \mathrm{U} / \mathrm{kg}$, i.p.). BGLs from blood samples taken from the tail vein using glucometer at $0,30,60$, and 120 min following the insulin injection. (38)

\subsection{Behavioral tests}

\subsubsection{Open field test (OFT)}

OFT is carried out to evaluate animal locomotor activity and anxiety. (39) The rats were taken inside the apparatus of OFT on the $13^{\text {th }}$ day of the study. OFT apparatus in which rats have previously unknown, was constructed of square based Plexiglas box ( $50 \times 50 \mathrm{~cm}$ with $25 \mathrm{~cm}$ walls). The ground is divided into twenty-five evenly spaced squares, each 10 $x 10 \mathrm{~cm}$ and consisted of two parts: the peripheral part (10 cm from each wall in the area) and central part. Each animal was separetly placed in the bottom right corner of the OFT apparatus and evaluated. The behavior of animals was recorded for 10 minutes using a video camera. Some parameters were evaluated such as the time spent in the central area, the number of squares passed, and the number of grooming and rearing of rats by two researchers as double-blind. The number of squares crossed by rats was considered as a measure of locomotor activity. The frequency of rearing was used to indicate discovery behavior. The time spent in the central area and the number of grooming was used to indicate the anxiety behavior of rats. The surface of apparatus were cleaned with $70 \%$ alcohol in consecutive trials to eliminate any bias that might have occurred due to odor of the previous rat.(40)

\subsubsection{Novel object recognition test (NORT)}

NORT is a widely used, relatively simple, and straightforward behavioral test for the examination of various aspects of learning and memory in rats. NORT is on the strength of spontaneous behavior of rodents to explore novelty with a naturally stimulating stimulus. When spontaneous behavior is examined, artificial stimulus, food deprivation, reinforcement and/or prior training are not required. NORT apparatus was constructed of black plexiglass open area (50 x $50 \mathrm{~cm}$ with $40 \mathrm{~cm}$ walls) in the dim light on day 14 (habituation) and 15 (test) of the study. Rats were habituated to an empty test area for 30 minutes to acclimate to the environment 24 hours before testing. NORT consisted of two three-minute test stage separated by an intertrial interval of one hour. Two identical objects were located in opposite corners for the first stage and these objects are now called familiar (F). During the second stage, one of the objects was replaced with a novel one $(\mathrm{N})$. The objects used in NORT differed by color, texture, and shape. NORT apparatus was cleaned after each rat with $70 \%$ alcohol to remove any urine or scent cues. 
Object placement was randomized for each test. Exploration of identical and novel objects was defined if rats licked, sniffed or touched the object. Based on the exploration time of each object, the discrimination index $[(\mathrm{N}-\mathrm{F}) /(\mathrm{N}+\mathrm{F})]$ and preferential index $[\mathrm{N} /(\mathrm{N}+\mathrm{F})]$ was calculated. Exploration time was scored by the treatments blind researcher. (41)

\subsubsection{Passive avoidance test (PAT)}

PAT is a classic fear-motivated test to examine long and shortterm memories in a relational manner. The test requires the animals to act opposite to their natural tendencies for the preference of dark areas and avoidance of bright ones. The test was carried out using an apparatus that comprises two compartments isolated with a retractable lid. One of the compartments is illuminated by a bright light, the other compartment is covered by dark opaque walls. Dark and light chambers are seperated by a guillotine door. The ground in both compartments is made of metal shocking grids. On the dark compartment, the ground is wired for 3 second to receive an electric shock of $0.5 \mathrm{~mA}$.

The acquisition and test stages of PAT were applied on day 16 and 17. On acquisition day, rats were placed in the bright compartment when the guillotine door was closed. At the end of 10-second period, the door was opened. After the rats moved into the dark compartment, the door was closed and electric shock of $0.5 \mathrm{~mA}$ was delivered via metal shocking grids. The first passage time of rat to the dark compartment was recorded. On day 17, the test stage was performed $24 \mathrm{~h}$ after the acquisition stage and it was similar to the previous stage with an exception that no electric shock was applied. The time spent for moving into the dark compartment was measured. In the test, a cut-off time was determined as 300 seconds if the animal did not enter the dark compartment. The experiment is terminated in this stiuation. Both compartments were cleaned with $70 \%$ ethanol after each rat tested.(40)

\subsubsection{Morris's water maze (MWM) test}

MWM was carried out to evaluate the spatial performance of the rats. MWM is consisting of two stages. MWM training was performed between day 18 and 21 and MWM prob test was performed on day 22. MWM apparatus comprised a round stainless steel tank (160 cm in diameter) with $40 \mathrm{~cm}$ walls filled with water $\left(23 \pm 1^{\circ} \mathrm{C}\right)$. The tank is divided into 4 quarters with 4 fixed points around it and there are different shapes and color 4 cues attached to the opposite of each direction. During all escape platform trials, a platform was submerged $1 \sim 1.5 \mathrm{~cm}$ under the water surface and this platform is the same color as the rest of the maze (to eliminate any false positives due to vision) in the middle of one of quarters of the area. Each trial commenced with the rats being released in the pool at one of the four main compass positions around the perimeter of the pool according to a pseudo-random sequence and allowed $75 \mathrm{~s}$ for finding the platform. If the rat could not find the escape platform within 75 seconds, it was gently directed to the platform and allowed to stay on it for 20 seconds. After a minute rats were placed in water to another direction. Thus, all rats were trained 16 times in 4 days, 4 different directions each day. The direction order was changed every day. Rats were subjected to learning, which was evaluated for reaching the platform. On day 5, 24 hours after the previous training session, the probe test in which the platform was removed from the tank and the rats were allowed to swim freely for 60 seconds was performed. The time to reach the target quadrant in the probe trial and the time spent in the target quadrant in both training and prob trials were recorded. Behaviors in the maze were monitored by a digital camera and the parameters were evaluated by two separate researchers.(42)

\subsection{Statistical Analysis}

All data were expressed as mean \pm standard error of mean (SEM). The results of the tests were analyzed with ANOVA followed by Tukey post-hoc test and represented as mean \pm S.E.M. $P$ values $<0.05$ were considered significant. Data analysis was performed using GraphPad Prism 6.5 software (San Diego, USA).

\section{RESULTS}

\subsection{Body weight}

There was no significant difference in b.w. between test groups for the first 4 weeks. After the injection of STZ, the difference in b.w. between the groups began to change significantly. However, the b.w. of $A D$ and $A D+C S$ group decreased significantly $(p<0.05)$ compared to the control group after 6 weeks. There was no significant difference between $C$ and $A D+M$ groups for 9 weeks.

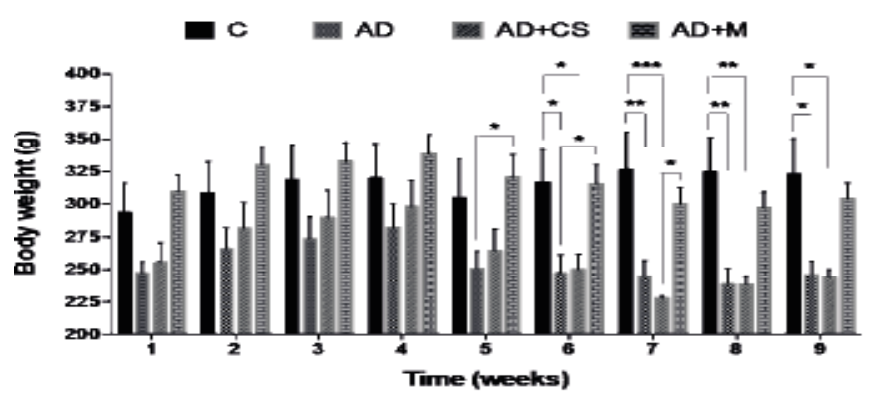

Figure 1. Body weight of rats in the period of 9 weeks. Each group $(n=12)$ represents Mean \pm SEM. ANOVA was performed followed by Tukey post hoc test. Values are represented statistically when *p $<0.05,{ }^{*} p<0.01, * * * p<0.001$ in comparison with eachother. (C: Control group, $A D$ : Alzheimer's disease group, $A D+C S$ : $C$. Sinensis treatment group, $A D+M$ : Metformin treatment group)

\subsection{Blood Glucose Level}

The glucose level of the control group was almost $100 \mathrm{mg} / \mathrm{dl}$ for the last 5 weeks of the experiment. Metformin treatment started to decrease BGL $(p<0.05)$ before $C$. Sinensis treatment compared to the control group as shown in Figure 2 on week 5. The blood glucose lowering effect of metformin continued until the end of the experiment. $C$. Sinensis treatment started 
significantly lowering BGL $(p<0.01)$ compared to the control group on week 8 and similar results were observed also on week 9. Metformin treatment tolerated BGL better than $C$. Sinensis treatment $(p<0.05)$ according to the results shown on weeks 8 and 9.

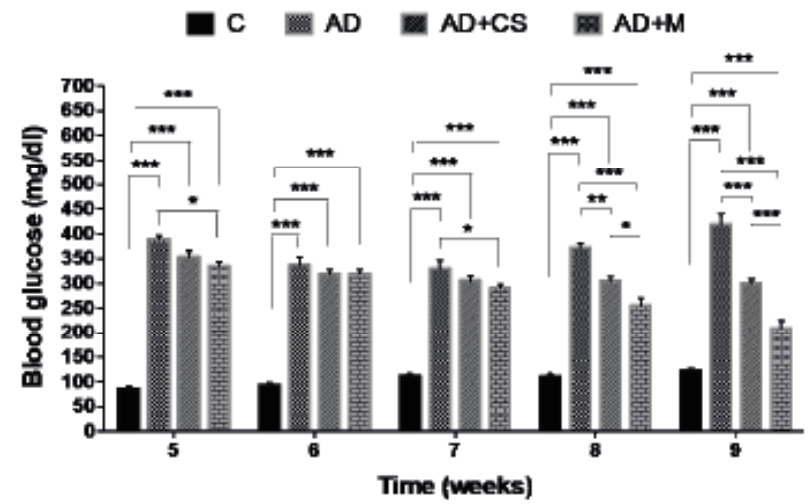

Figure 2. Blood glucose level of rats after starting the treatment. Each group $(n=12)$ represents Mean \pm SEM. ANOVA was performed followed by Tukey post hoc test. Values are represented statistically when ${ }^{*} p<0.05,{ }^{*} p<0.01,{ }^{* *} p<0.001$ in comparison with eachother. (C: Control group, AD: Alzheimer's disease group, $A D+C S$ : $C$. Sinensis treatment group, $A D+M$ : Metformin treatment group)

\subsection{Oral glucose tolerance test (OGTT)}

To determine the effect of $C$. Sinensis on glucose tolerance in rats, OGTT was applied (Figure 3). The slope of the curve of blood glucose was significantly higher in the non-treated rats than treated rats during the first $30 \mathrm{~min}$. The slope of the curve was lower in the $A D+M$ group than the $A D+C S$ group due to the ability of Metformin to better tolerate glucose. As shown in Figure 3, metformin better tolerated blood glucose levels from $30 \mathrm{~min}$ to $90 \mathrm{~min}$ compared to $C$. Sinensis ( $\mathrm{p}$ $0.01)$ and control group $(p<0.001)$. Whereas, $C$. Sinensis treatment demonstrated better glucose tolerance only at 60 min compared to the control group $(p<0.05)$.

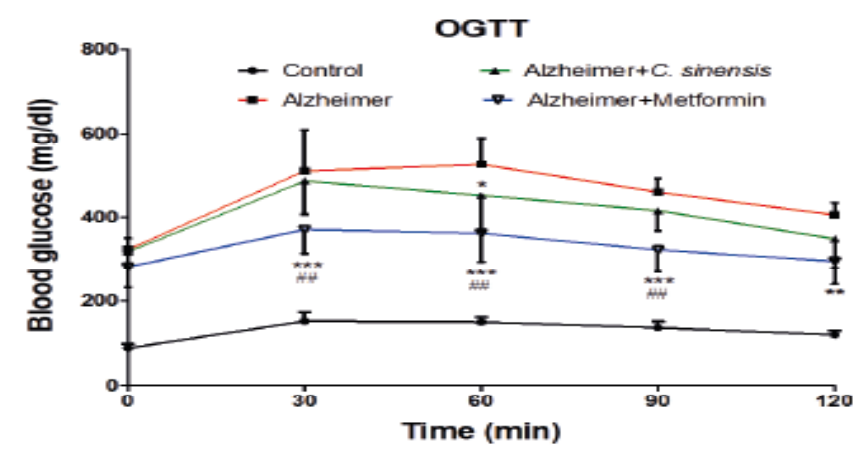

Figure 3. Oral glucose tolerance test in rats. Each group $(n=12)$ represents Mean \pm SEM. ANOVA was performed followed by Tukey post hoc test. ${ }^{*} p<0.05,{ }^{* *} p<0.01,{ }^{* * *} p<0.001$ represent statistical differences of metformin and $C$. Sinensis groups compared to Alzheimer group and $\# p<0.05, \# \# p<0.01, \# \# \# p<0.001$ represent statistical differences of metformin group compared to $C$. Sinensis group.

\subsection{Insulin tolerance test (ITT)}

As shown in Figure 4, an ideal insulin tolerance was observed in the control group. BGL decreased significantly for $60 \mathrm{~min}$ in healthy rats in a similar manner to other groups but BGL was well tolerated by the healthy body. Whereas, BGL was not tolerated by the non-treated Alzheimer group and was not well tolerated with the AD+CS group. The slope of the curve belongs to control and $A D+C S$ groups were similar until $90 \mathrm{~min}$ and the slope decreased for the AD+CS group after 90 min but there is no significant difference between $A D$ and $A D+C S$ group in $120 \mathrm{~min}$. When we compared the $A D+C S$ group with the $A D+M$ group, it is clearly seen that Metformin tolerated BGL significantly better than $C$. Sinensis according to $120 \mathrm{~min}(\mathrm{p}<0.05)$.

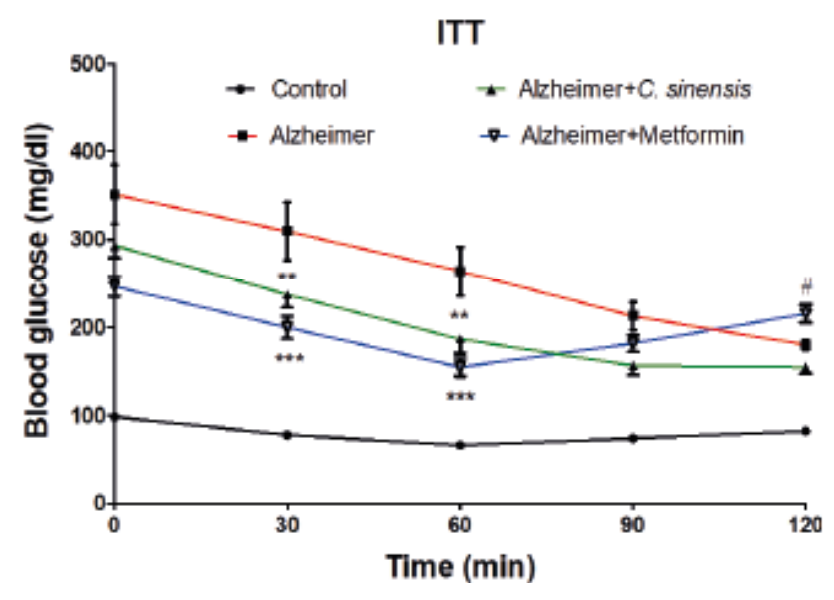

Figure 4. Insulin tolerance test in rats. Each group $(n=12)$ represents Mean \pm SEM. ANOVA was performed followed by Tukey post hoc test. ${ }^{*} p<0.05, * * p<0.01, * * * p<0.001$ represent statistical differences of metformin and $C$. Sinensis groups compared to Alzheimer group and $\# p<0.05, \# \# p<0.01, \# \# \# p<0.001$ represent statistical differences of metformin group compared to $C$. Sinensis group.

\subsection{Behavioral tests}

\subsubsection{Open field test}

\subsubsection{Crossing activity}

As shown in Figure $5 \mathrm{~A}$, the number of square crosses of rats were measured to evaluate locomotor activity. There is no significant difference between treatment groups on squares crossed in OFT. The number of square crosses significantly decreased in the AD group compared to the control group $(p<0.05)$ and increased in the treatment groups compared to the AD group but there is no significant difference.

\subsubsection{Rearing activity}

The number of rearings of rats were measured to evaluate exploratory behavior. The number of rearings significantly decreased in the $A D, A D+C S$, and $A D+M$ groups compared 
to the control group $(p<0.05)$ and there is no significant difference between treatment gorups and the $A D$ group (Figure 5B).

\subsubsection{Latency to enter center}

The latency to enter center was measured in OFT. Latency to enter the center zone of the open-field was not found to be altered significantly between treatment groups. It considerably decreased in $A D$ and $A D+C S$ groups compared to the control group $(p<0.05)$ but it was similar to the control group in the $A D+M$ group (Figure $5 C$ ).
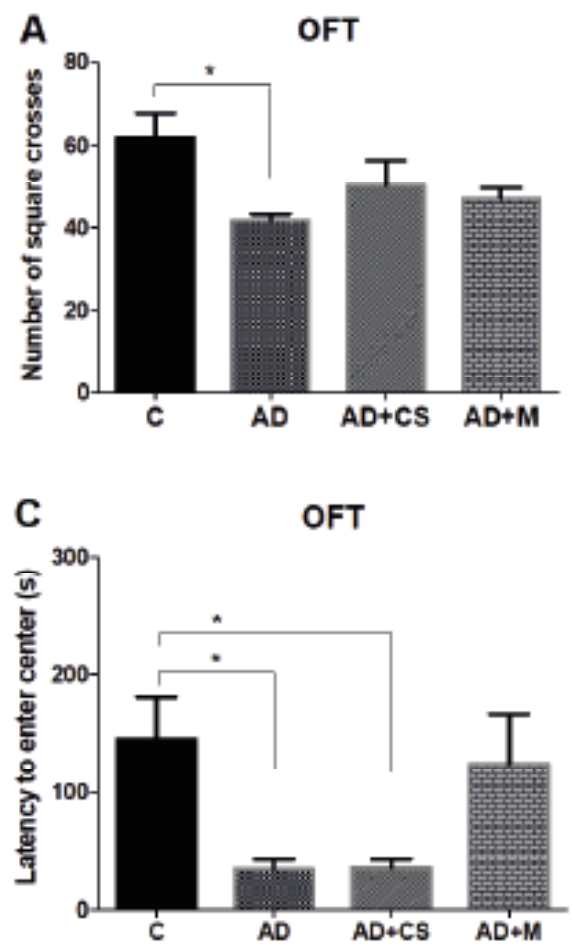

\subsubsection{Grooming activity}

The number of grooming of rats was counted as a measure of anxiety (Figure 5D). The number of rearings significantly decreased in the non-treated Alzheimer group compared to the control group $(p<0.05)$ and increased in the treatment groups compared to the $A D$ group but there is no significant difference.

\subsubsection{Time spent in the central zone}

The time spent in the central zone was also evaluated as a measure of anxiety. There is no significant difference between groups (Figure 5E).

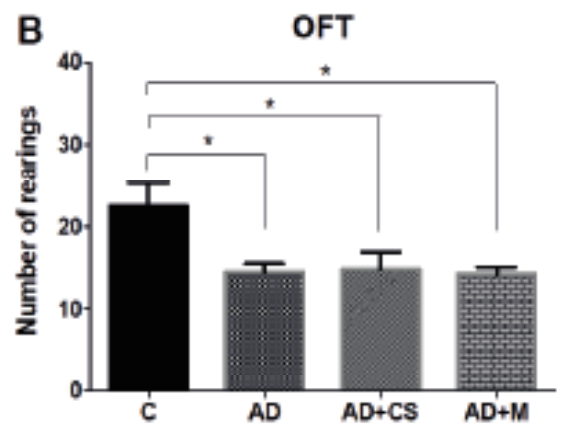

D OFT

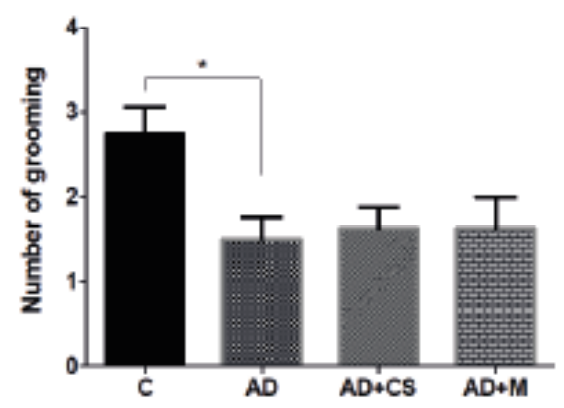

OFT

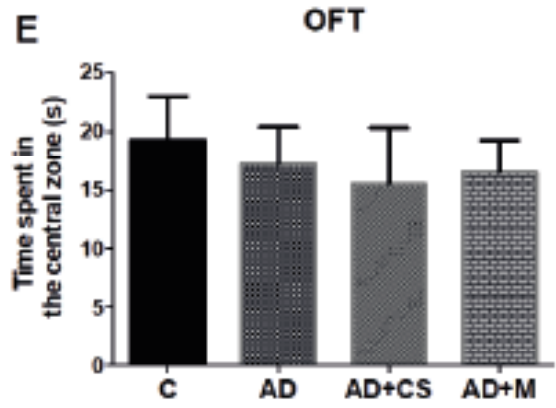

Figure 5. (A) The numer of square crosses, $(B)$ the number of rearings, $(C)$ latency to enter center, $(D)$ the number of grooming, and $(E)$ time spent in the central zone of rats in OFT. Each group $(n=12)$ represents Mean \pm SEM. ANOVA was performed followed by Tukey post hoc test. * $p$ $<0.05,{ }^{*} p<0.01,{ }^{* *} p<0.001$ in comparison with eachother. (C: Control group, AD: Alzheimer's disease group, AD+CS: C. Sinensis treatment group, $A D+M$ : Metformin treatment group)

\subsubsection{Novel object recognition test}

NORT was used for evaluating short-term memory. In NORT, the object recognition was performed between the training phase and the test phase at 1-hour intervals. There are significant differences between the exploration time of familiar and novel object in control ( $p<0.001)$, AD+CS ( $p<$ $0.05)$, and $A D+M$ groups $(p<0.05)$ but there is no significant difference in $A D$ group (Figure 6). 


\section{NORT}

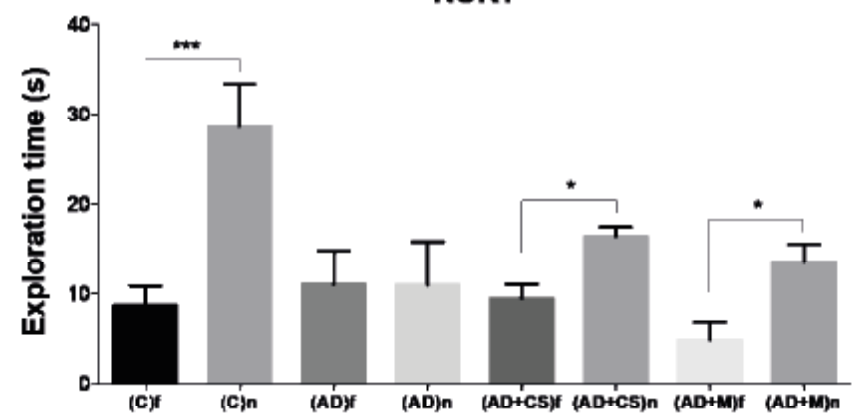

Figure 6. Exploration time of rats in NORT. Each group $(n=12)$ represents Mean \pm SEM. ANOVA was performed followed by Tukey post hoc test. ${ }^{*} p<0.05,{ }^{* *} p<0.01,{ }^{* * *} p<0.001$ in comparison with eachother. ((C)f: Control group with familiar object, (C)n: Control group with familiar object, $(A D) f$ : Alzheimer's disease group with famailiar object, $(A D)$ n: Alzheimer's disease group with novel object, $A D$ : Alzheimer's disease group, $A D+C S$ : $C$. Sinensis treatment group, $A D+M$ : Metformin treatment group)

Discrimination and preferential index were also evaluated in NORT and it is clearly seen that all groups are significantly higher $(p<0.05)$ than $A D$ group in discrimination index (Figure 7A). $C$ and $A D+M$ groups had significantly higher preferential index compared AD group (Figure 7B). Short-term memory impairment was improved with metformin and $C$. Sinensis treatments.
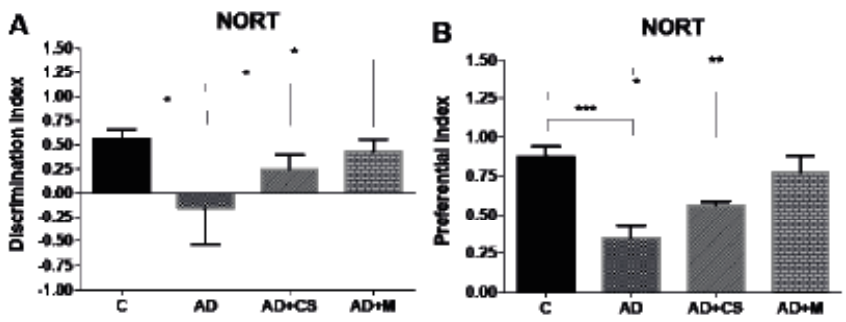

Figure 7. (A) Discrimination index and (B) preferential index of rats in NORT. Each group $(n=6)$ represents Mean \pm SEM. ANOVA was performed followed by Tukey post hoc test. ${ }^{*} p<0.05,{ }^{* *} p<0.01$, ${ }^{* * *} p<0.001$ in comparison with eachother. (C: Control group, AD: Alzheimer's disease group, $A D+C S$ : $C$. Sinensis treatment group, $A D+M$ : Metformin treatment group)

\subsubsection{Passive avoidance test}

The step-through latency (STL) of rats was measured to evaluate long and short-term memories in a relational manner. The STL time decreased in AD group compared to $C$ group. The treatments increased STL time compared to $A D$ group but there was no significant difference between them.

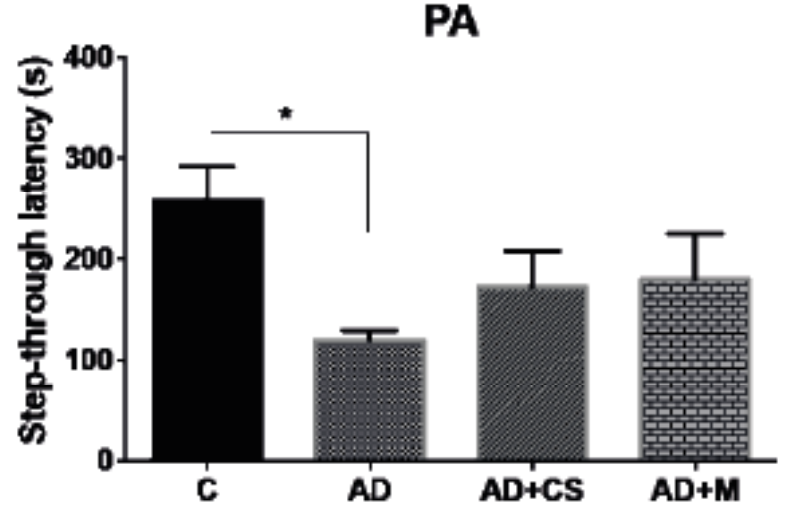

Figure 8. Step-through latency of rats in PA test. Each group $(n=6)$ represents Mean \pm SEM. ANOVA was performed followed by Tukey post hoc test. ${ }^{*} p<0.05,{ }^{* *} p<0.01,{ }^{* * *} p<0.001$ in comparison with eachother. (C: Control group, $A D$ : Alzheimer's disease group, $A D+C S$ : $C$. Sinensis treatment group, $A D+M$ : Metformin treatment group)

\subsubsection{Morris's Water Maze Test}

Probe test was performed 24 hours after the last training day. In this test, the total time spent and the time to reach the target quadrant where the platform is located was observed. AD group reached the target quadrant later than control group $(p<0.05)$ in all days and the total time spent in the quadrant decreased. When the treatment groups were examined, it was observed that $A D+M$ group significantly improved it compared to AD+CS group on day $4(p<0.01)$.

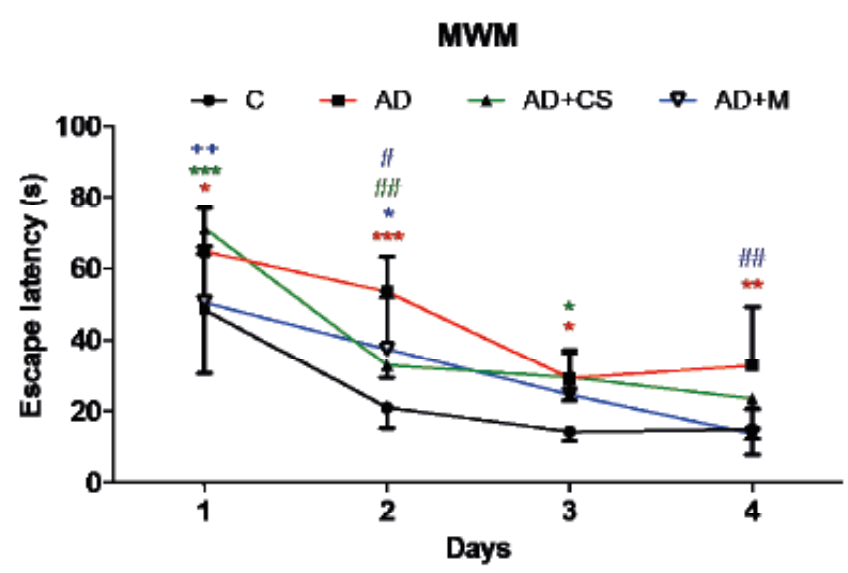

Figure 9. Latency to platform of rats in MWM test. Each group ( $n$ = 6) represents Mean \pm SEM. ANOVA was performed followed by Tukey post hoc test. ${ }^{*} p<0.05,{ }^{* *} p<0.01,{ }^{* * *} p<0.001$ represent statistical differences compared to C group and \#p<0.05, \#\#p< 0.01 , \#\#\#p $<0.001$ represent statistical differences compared to $A D$ group. $+p<0.05,++p<0.01,+++p<0.001$ represent statistical differences compared to $A D+C S$ group. ( $C$ : Control group, $A D$ : Alzheimer's disease group, $A D+C S$ : $C$. sinensis treatment group, $A D+M$ : Metformin treatment group) 


\section{DISCUSSION}

$A D$ is an irreversible disorder destroys memory and thinking skills and it is estimated that at least 50 million are living with AD or other dementias (43). T2DM is a chronic disease characterized by high levels of glucose in the blood and affecting over 300 million people worldwide (44). When diabetes is not controlled well, BGLs rise and stay high for days to weeks. Within this period, most organs including the brain will be damaged and researchers have found many pieces of evidence that T2DM could link to $A D$, which is the most common form of dementia. Moreover, epidemiological studies have shown that there are pathophysiological connections between T2DM and AD. Insulin resistance majorly affects the disturbance of various biological processes and signaling pathways according to the recent evidences (45). Therefore, HFD/STZ-treated rat models were used to provide T2DM-induced AD-like alterations in our study. HFD is one of the most common risk factor for T2DM and $A D$, thus it has been chosen for inducing T2DM. HFD is also mimicking a real human diet, which causes insulin resistance and then type 2 diabetes to occur (46). It has been stated that diet-induced diabetes models represent a more true mechanism of DM pathogenesis rather than genetic factors (47). In literature, this model has been used in many studies in the evaluation of treatments for the most type of dementia (48).

Current pharmacological treatment for AD with antidementia drugs, which are cholinesterase inhibitors and memantine, have limited properties such as temporary and symptomatic support to cognitive functions. Substantial funding has been dedicated to the development of new pharmaceutical compounds with disease-modifying properties over the past decade (49). Besides, traditional medicine presents different plant-derived lead molecules that may be beneficial for further medical research. Traditional medicines are consumed as a memory enhancer for centuries and they have been used in the treatment of memory deficits such as dementia, amnesia, and AD (50). Green tea (C. sinensis) is one of these traditional medicines which are used in the treatment of neurodegenerative diseases such as Alzheimer's and Parkinson's disease (51).

SGLT inhibitors are currently being investigated as the medication in AD (52). In our study, $C$. sinensis was chosen due to its SGLT-1 inhibitor effects and T2DM-induced AD model was chosen to observe its anti-Alzheimer effects. The antidiabetic potential of $C$. sinensis with OGTT and ITT; and mainly its anti-Alzheimer effect with behavioral tests such as open field, novel object recognition, passive avoidance, and Morris's water maze test was investigated.

OGTT is currently the gold standard for the diagnosis of diabetes. Our results are in accordance with what showed that $C$. sinensis hydroalcoholic extract slighlty decreased the BGL of diabetic rats in $60 \mathrm{~min}$. It is clearly seen that $C$. sinensis tolerated BGL for a short time but metformin, the first medication prescribed for T2DM, tolerated BGL during the test for $120 \mathrm{~min}$.
In the present study, HFD/STZ-treated rat model occurred a significant memory deficit according to the learning and memory tests, which are OFT, NORT, PAT, and MWM. The cognition-enhancing activity of drugs is assessed by NORT and PAT, which are a relatively high-throughput, robust, and sensitive tests. MWM test is used to analyze spatial memory by measuring the escape latency time to reach a hidden platform. Locomotor activity, exploratory behavior, and anxiety was measured by OFT (53-55).

Animal behaviors such as the number of square crosses, frequency of rearing activity, latency to enter center, frequency of grooming activity, and time spent in the central zone are used as measures of locomotor activity and exploration. An increase in these parameters indicates a rise in locomotion and exploration (56). According to the results, $C$. sinensis increased the number of square crosses and the frequency of grooming activity in a similar manner to metformin.

The NORT evaluates the natural propensity of a rat to explore a novel versus a familiar object. This test provides to gain information about two different components, which are exploratory behavior and memory retention. Hence, the rats should sufficiently explore the familiar object during the pretest phase and then distinguish it with the new one in the test phase (57). The exploration of the novel object was reduced in HFD/STZ-treated rat model. It was also shown in the discrimination index in the NORT and HFD/STZtreated rats demonstrated decreased exploratory behavior. In contrast, metformin and $C$. sinensis treatments improved these parameters.

The PAT is connected with the amygdala and evaluates emotional memory. PAT is associated with long-term or reference memory and it is for studying learning and memory after a stressful stimulus (58). The step-through latency decreased in HFD/STZ-treated rat model but it improved with metformin and $C$. sinensis treatment.

MWM test was performed to rats for evaluating spatial learning (59). There was a decrease in latency to find the platform underwater in all groups during the training phase. These results show that all rats learned the platform. However, it was clearly seen that AD group needs more time for training. These results show that there is a deterioration in the coding and remembering of the spatial memory. According to the results obtained by the MWM test, metformin treatment improved learning.

\section{CONCLUSIONS}

C. sinensis was used in the treatment of T2DM-induced ADlike alterations via its antidiabetic effects. OGTT and ITT were performed to evaulate its antidiabetic effects and also OFT, NORT, PAT, and MWM were performed to investigate its antiAlzheimer effects. $C$. sinensis improved short-term memory and increased the locomotor activity in rats according to the results obtained by NORT, OFT, and PAT. 
Conflict of Interest: The authors have no conflict of interest to declare.

Acknowledgements: This study was supported by Marmara University, Scientific Research Projects Committee (MUBAPKO; SAG-K-170.118.0001).

\section{REFERENCES}

[1] Swerdlow RH. Pathogenesis of Alzheimer's disease. Clin Interv Aging 2007;2:347-359.

[2] Butterfield DA. Phosphoproteomics of Alzheimer disease brain: Insights into altered brain protein regulation of critical neuronal functions and their contributions to subsequent cognitive loss. Biochimica et Biophysica Acta (BBA) - Molecular Basis of Disease 2019;1865:2031-2039.

[3] Association As. 2019 Alzheimer's disease facts and figures. Alzheimer's \& Dementia 2019;15:321-387.

[4] Liang D, Lu H. Salivary biological biomarkers for Alzheimer's disease. Archives of Oral Biology 2019;105:5-12.

[5] Kocahan S, Doğan Z. Mechanisms of Alzheimer's Disease Pathogenesis and Prevention: The Brain, Neural Pathology, N-methyl-D-aspartate Receptors, Tau Protein and Other Risk Factors. Clin Psychopharmacol Neurosci 2017;15:1-8.

[6] Calderon-Garcidueñas AL, Duyckaerts C. Chapter 23 Alzheimer disease. In: Kovacs GG, Alafuzoff I, editors. Handbook of Clinical Neurology: Elsevier; 2018. p. 325-337.

[7] Beyer L, Schnabel J, Kazmierczak P, Ewers M, Schönecker S, Prix C, Meyer-Wilmes J, Unterrainer M, Catak C, Pogarell O, Perneczky R, Albert NL, Bartenstein P, Danek A, Buerger K, Levin J, Rominger A, Brendel M. Neuronal injury biomarkers for assessment of the individual cognitive reserve in clinically suspected Alzheimer's disease. Neurolmage: Clinical 2019;24:101949.

[8] Albensi BC. Chapter Two - Dysfunction of mitochondria: Implications for Alzheimer's disease. In: Fernyhough P, Calcutt NA, editors. International Review of Neurobiology: Academic Press; 2019. p. 13-27.

[9] Veljkovic E, Xia W, Phillips B, Wong ET, Ho J, Oviedo A, Hoeng J, Peitsch M. Chapter 2 - Alzheimer's Disease. In: Veljkovic E, Xia W, Phillips B, Wong ET, Ho J, Oviedo A, Hoeng J, Peitsch $M$, editors. Nicotine and Other Tobacco Compounds in Neurodegenerative and Psychiatric Diseases: Academic Press; 2018. p. 13-23.

[10] Green RC, Cupples LA, Go R, Benke KS, Edeki T, Griffith PA, Williams M, Hipps Y, Graff-Radford N, Bachman D, Farrer LA. Risk of dementia among white and African American relatives of patients with Alzheimer disease. Jama 2002;287:329-336.

[11] Hampel H, Mesulam MM, Cuello AC, Farlow MR, Giacobini E, Grossberg GT, Khachaturian AS, Vergallo A, Cavedo E, Snyder PJ, Khachaturian ZS. The cholinergic system in the pathophysiology and treatment of Alzheimer's disease. Brain 2018;141:1917-1733.

[12] Ding F, Yao J, Rettberg JR, Chen S, Brinton RD. Early Decline in Glucose Transport and Metabolism Precedes Shift to Ketogenic System in Female Aging and Alzheimer's Mouse Brain: Implication for Bioenergetic Intervention. PLOS ONE 2013;8:e79977.

[13] Cam ME, Hazar-Yavuz AN, Yildiz S, Ertas B, Ayaz Adakul B, Taskin T, Alan S, Kabasakal L. The methanolic extract of Thymus praecox subsp. skorpilii var. skorpilii restores glucose homeostasis, ameliorates insulin resistance and improves pancreatic $\beta$-cell function on streptozotocin/nicotinamideinduced type 2 diabetic rats. Journal of Ethnopharmacology 2019;231:29-38.

[14] Cam ME, Yildiz S, Ertas B, Acar AE, Taskin T, Kabasakal L. Antidiabetic effects of Salvia triloba and Thymus praecox subsp. skorpilii var. skorpilii in a rat model of streptozotocin/ nicotinamide-induced diabetes. Marmara Pharmaceutical Journal 2017;21:818-827.

[15] Mushtaq G, Khan JA, Kamal MA. Biological mechanisms linking Alzheimer's disease and type-2 diabetes mellitus. CNS \& neurological disorders drug targets 2014;13:1192-1201.

[16] Yarchoan M, Arnold SE. Repurposing Diabetes Drugs for Brain Insulin Resistance in Alzheimer Disease. Diabetes 2014;63:2253.

[17] Kang $\mathrm{S}$, Kim CH, Jung $\mathrm{H}$, Kim E, Song HT, Lee JE. Agmatine ameliorates type 2 diabetes induced-Alzheimer's disease-like alterations in high-fat diet-fed mice via reactivation of blunted insulin signalling. Neuropharmacology 2017;113:467-479.

[18] Magalhaes DA, Kume WT, Correia FS, Queiroz TS, Allebrandt Neto EW, Santos MPD, Kawashita NH, Franca SA. High-fat diet and streptozotocin in the induction of type 2 diabetes mellitus: a new proposal. Anais da Academia Brasileira de Ciencias 2019;91:e20180314.

[19] Skovs $\varnothing$ S. Modeling type 2 diabetes in rats using high fat diet and streptozotocin. J Diabetes Investig 2014;5:349-358.

[20] Chauhdary Z, Saleem U, Ahmad B, Shah S, Shah MA. Neuroprotective evaluation of Tribulus terrestris L. in aluminum chloride induced Alzheimer's disease. Pakistan journal of pharmaceutical sciences 2019;32:805-816.

[21] Zhang X, Wang X, Hu X, Chu X, Li X, Han F. Neuroprotective effects of a Rhodiola crenulata extract on amyloid-beta peptides (Abeta1-42) - induced cognitive deficits in rat models of Alzheimer's disease. Phytomedicine : international journal of phytotherapy and phytopharmacology 2019;57:331-338.

[22] Raheja S, Girdhar A, Kamboj A, Lather V, Pandita D. Aegle marmelos leaf extract ameliorates the cognitive impairment and oxidative stress induced by intracerebroventricular streptozotocin in male rats. Life sciences 2019;221:196203.

[23] Wagle A, Seong SH, Shrestha S, Jung HA, Choi JS. Korean Thistle (Cirsium japonicum var. maackii (Maxim.) Matsum.): A Potential Dietary Supplement against Diabetes and Alzheimer's Disease. Molecules (Basel, Switzerland) 2019;24.

[24] Berg-Weger M, Stewart DB. Non-Pharmacologic Interventions for Persons with Dementia. Mo Med 2017;114:116-119.

[25] Koenig AM, Mechanic-Hamilton D, Xie SX, Combs MF, Cappola AR, Xie L, Detre JA, Wolk DA, Arnold SE. Effects of the Insulin Sensitizer Metformin in Alzheimer Disease: Pilot Data From a Randomized Placebo-controlled Crossover Study. Alzheimer Dis Assoc Disord 2017;31:107-113.

[26] Suraphad P, Suklaew PO, Ngamukote S, Adisakwattana S, Mäkynen K. The Effect of Isomaltulose Together with Green Tea on Glycemic Response and Antioxidant Capacity: A Single-Blind, Crossover Study in Healthy Subjects. Nutrients 2017;9:464.

[27] Li H, Wu X, Wu Q, Gong D, Shi M, Guan L, Zhang J, Liu J, Yuan B, Han G, Zou Y. Green tea polyphenols protect against okadaic acid-induced acute learning and memory impairments in rats. Nutrition (Burbank, Los Angeles County, Calif) 2014;30:337-342. 
[28] Snoussi C, Ducroc R, Hamdaoui MH, Dhaouadi K, Abaidi H, Cluzeaud F, Nazaret C, Le Gall M, Bado A. Green tea decoction improves glucose tolerance and reduces weight gain of rats fed normal and high-fat diet. The Journal of nutritional biochemistry 2014;25:557-564.

[29] Shivashankara AR, Kumar A, Ravi R, Simon P, Rai P, Francis A, Baliga MS. Chapter 10 - Use of Tea (Camellia sinensis [L.] Kuntze) as a Hepatoprotective Agent in Geriatric Conditions. In: Watson RR, editor. Foods and Dietary Supplements in the Prevention and Treatment of Disease in Older Adults. San Diego: Academic Press; 2015. p. 99-104.

[30] Al-Attar AM, Zari TA. Influences of crude extract of tea leaves, Camellia sinensis, on streptozotocin diabetic male albino mice. Saudi J Biol Sci 2010;17:295-301.

[31] Pervin M, Unno K, Ohishi T, Tanabe H, Miyoshi N, Nakamura Y. Beneficial Effects of Green Tea Catechins on Neurodegenerative Diseases. Molecules (Basel, Switzerland) 2018;23:1297.

[32] Yang Z, Xu Y, Jie G, He P, Tu Y. Study on the antioxidant activity of tea flowers (Camellia sinensis). Asia Pacific journal of clinical nutrition 2007;16 Suppl 1:148-152.

[33] Bedrood Z, Rameshrad M, Hosseinzadeh H. Toxicological effects of Camellia sinensis (green tea): A review. Phytotherapy research : PTR 2018;32:1163-1180.

[34] Prasanth MI, Sivamaruthi BS, Chaiyasut C, Tencomnao T. A Review of the Role of Green Tea (Camellia sinensis) in Antiphotoaging, Stress Resistance, Neuroprotection, and Autophagy. Nutrients 2019;11.

[35] Ide K, Matsuoka N, Yamada H, Furushima D, Kawakami K. Effects of Tea Catechins on Alzheimer's Disease: Recent Updates and Perspectives. Molecules (Basel, Switzerland) 2018;23:2357.

[36] Sharangi AB. Medicinal and therapeutic potentialities of tea (Camellia sinensis L.) - A review. Food Research International 2009;42:529-535.

[37] Asadbegi M, Yaghmaei P, Salehi I, Ebrahim-Habibi A, Komaki A. Neuroprotective effects of metformin against $A \beta$-mediated inhibition of long-term potentiation in rats fed a high-fat diet. Brain Research Bulletin 2016;121:178-185.

[38] Boland B, Mumphrey MB, Hao Z, Gill B, Townsend RL, Yu S, Münzberg $\mathrm{H}$, Morrison $\mathrm{CD}$, Trevaskis JL, Berthoud H-R. The PYY/Y2R-Deficient Mouse Responds Normally to High-Fat Diet and Gastric Bypass Surgery. Nutrients 2019;11:585.

[39] Zimcikova E, Simko J, Karesova I, Kremlacek J, Malakova J. Behavioral effects of antiepileptic drugs in rats: Are the effects on mood and behavior detectable in open-field test? Seizure 2017;52:35-40.

[40] Abdel-Aal RA, Assi AA, Kostandy BB. Rivastigmine reverses aluminum-induced behavioral changes in rats. European journal of pharmacology 2011;659:169-176.

[41] Smith BM, Yao X, Chen KS, Kirby ED. A Larger Social Network Enhances Novel Object Location Memory and Reduces Hippocampal Microgliosis in Aged Mice. Frontiers in aging neuroscience 2018;10:142.

[42] Vorhees CV, Williams MT. Morris water maze: procedures for assessing spatial and related forms of learning and memory. Nat Protoc 2006;1:848-858.

[43] Browne D, McGuinness B, Woodside JV, McKay GJ. Vitamin E and Alzheimer's disease: what do we know so far? Clin Interv Aging 2019;14:1303-1317.
[44] Sami W, Ansari T, Butt NS, Hamid MRA. Effect of diet on type 2 diabetes mellitus: A review. Int J Health Sci (Qassim) 2017;11:65-71.

[45] Mittal K, Katare DP. Shared links between type 2 diabetes mellitus and Alzheimer's disease: A review. Diabetes \& Metabolic Syndrome: Clinical Research \& Reviews 2016;10:S144-S9.

[46] Moreno-Fernández S, Garcés-Rimón M, Vera G, Astier J, Landrier JF, Miguel M. High Fat/High Glucose Diet Induces Metabolic Syndrome in an Experimental Rat Model. Nutrients 2018;10:1502.

[47] Zhuo J, Zeng Q, Cai D, Zeng X, Chen Y, Gan H, Huang X, Yao N, Huang $D$, Zhang $C$. Evaluation of type 2 diabetic mellitus animal models via interactions between insulin and mitogen-activated protein kinase signaling pathways induced by a high fat and sugar diet and streptozotocin. Mol Med Rep 2018;17:5132-5142.

[48] Kang S, Kim C-H, Jung H, Kim E, Song H-T, Lee JE. Agmatine ameliorates type 2 diabetes induced-Alzheimer's disease-like alterations in high-fat diet-fed mice via reactivation of blunted insulin signalling. Neuropharmacology 2017;113:467-479.

[49] Aprahamian I, Stella F, Forlenza OV. New treatment strategies for Alzheimer's disease: is there a hope? Indian J Med Res 2013;138:449-460.

[50] Akram M, Nawaz A. Effects of medicinal plants on Alzheimer\&\#39;s disease and memory deficits. Neural Regeneration Research 2017;12:660-670.

[51] Prasanth MI, Sivamaruthi BS, Chaiyasut C, Tencomnao T. A Review of the Role of Green Tea (Camellia sinensis) in Antiphotoaging, Stress Resistance, Neuroprotection, and Autophagy. Nutrients 2019;11:474.

[52] Simes BC, MacGregor GG. Sodium-Glucose Cotransporter-2 (SGLT2) Inhibitors: A Clinician's Guide. Diabetes Metab Syndr Obes 2019;12:2125-2136.

[53] Rinwa P, Jaggi AS, Singh N. Pharmacological investigation of memory restorative effect of riluzole in mice. Indian J Pharmacol 2012;44:366-371.

[54] Mathiasen JR, DiCamillo A. Novel object recognition in the rat: a facile assay for cognitive function. Curr Protoc Pharmacol 2010; Chapter 5:5.59.

[55] Terry AV. Spatial Navigation (Water Maze) Tasks. 2nd ed: CRC Press/Taylor \& Francis, Boca Raton (FL); 2009.

[56] Arika WM, Kibiti CM, Njagi JM, Ngugi MP. Effects of DCM Leaf Extract of Gnidia glauca (Fresen) on Locomotor Activity, Anxiety, and Exploration-Like Behaviors in High-Fat DietInduced Obese Rats. Behav Neurol 2019;2019:7359235.

[57] Foyet HS, Abaïssou HHN, Wado E, Acha EA, Alin C. Emilia coccinae (SIMS) G Extract improves memory impairment, cholinergic dysfunction, and oxidative stress damage in scopolamine-treated rats. BMC Complement Altern Med 2015;15:333.

[58] Wang J, Wang X, Lv B, Yuan W, Feng Z, Mi W, Zhang H. Effects of Fructus Akebiae on learning and memory impairment in a scopolamine-induced animal model of dementia. Exp Ther Med 2014;8:671-675.

[59] Yildiz S, Cam ME, Keles R, Hazar-Yavuz AN, Kabasakal L. A PPARalpha agonist gemfibrozil ameliorates cognitive and memory impairments in a sporadic Alzheimer's disease rat model. European Neuropsychopharmacology 2019;29:S263-S4. 
